\title{
PROPRIEDADES FÍSICO-QUÍMICAS DA FIBRA EM DETERGENTE NEUTRO DE DIFERENTES PALHAS DE TRIGO
}

\section{(Physico-chemical properties of fibers from wheat straws in neural detergent)}

\author{
WARPECHOWSKI, M.B. ${ }^{1}$; PINHEIRO, C.C. ${ }^{2}$; CIOCCA, M.L.S. ${ }^{3}$
}

1Departamento de Zootecnia, Universidade Federal do Paraná); ${ }^{2}$ Mestranda do Curso de Pós Graduação em Ciências Veterinárias/UFPR; ${ }^{3}$ Médica Veterinária, Departamento de Zootecnia, Universidade Federal do Rio Grande do Sul.

\begin{abstract}
RESUMO - Foram avaliadas as propriedades físicoquímicas de capacidade tamponante e de hidratação da fibra em detergente neutro de cinco amostras de palha de quatro variedades de trigo. Foi utilizado delineamento em blocos casualizados (corridas laboratoriais), com seis repetições de cada material. A capacidade tamponante, medida através da acidez titulável (mmol HCl/100g matéria seca de fibra), foi significativamente maior para BR-23B $(61,2)$, BR-35 $(60,2)$ e CEP-19 $(59,3)$, e menor para BR-23A $(53,4)$ e CEP-21 $(52,2)(P<0,05)$. A diferença entre as palhas foi maior no intervalo de $\mathrm{pH}$ entre 5,4 e 2,7, e a acidez titulável medida neste intervalo foi mais sensível, diferenciando as amostras BR23B e CEP19 $(P<0,05)$. Não houve diferença significativa entre as palhas para a capacidade de hidratação (média $=5,34 \mathrm{~g} \mathrm{H}_{2} \mathrm{O} / \mathrm{g}$ matéria seca de fibra, $P>0,05)$, embora esta medida tenha apresentado correlação positiva com a acidez titulável $(r=0,88, P<0,05)$. A capacidade tamponante da fibra de palhas de trigo parece depender tanto da variedade quanto da partida da amostra.
\end{abstract}

Palavras-chave: capacidade de hidratação, capacidade tamponante, fibra em detergente neutro, palha de trigo, propriedades físico-químicas.

ABSTRACT - The physco-chemical properties of buffering capacity and water-holding capacity were evaluated in the straw's neutral-detergent fiber from five samples of four wheat varieties. A block randomized design was utilized (laboratory batch as blocks), with six replicates for each material. The buffering capacity, measured by the titrable acidity (mmol HCl/100g fiber dry matter), was higher for BR-23B (61.2), BR-35 (60.2) and CEP-19 (59.3), and smaller for BR-23A (53.4) and CEP-21 (52.2) $(\mathrm{P}<0.05)$. The difference among straws was higher in the $\mathrm{pH}$ range of 5.4 to 2.7 , and the titrable acidity measured in that range was more sensible, detecting the difference between the samples of BR23B and CEP19 $(P<0.05)$. There was no significant difference among straws for the water-holding capacity (average $=5.34 \mathrm{~g} \mathrm{H}_{2} \mathrm{O} / \mathrm{g}$ fiber dry matter, $\mathrm{P}>0.05$ ), however that measure showed positive correlation with the titrable acidity $(r=0.88, P<0.05)$. The buffering capacity in wheat straw fiber's seems to be dependent to the variety as well as the source of the sample.

Key-words: buffering capacity, neutral-detergent fiber, physico-chemical properties, water-holding capacity, wheat straw.

\section{Introdução}

Os efeitos causados pela fração fibrosa presente nas dietas animais sobre os processos de fermentação, digestão e absorção de nutrientes no trato gastrintestinal variam de acordo com o nível de fibra e também com as propriedades físico-químicas dessa fração. E essas propriedades físico-químicas são dependentes da fonte da fibra, de sua composição química e de sua estrutura macromolecular (JERACI e HORVATH, 1989; VAN SOEST et al., 1991; ANNISON e CHOCT, 1994; FERREIRA, 1994).

Algumas propriedades físico-químicas da fração insolúvel da fibra como a capacidade tamponante e a capacidade de hidratação têm sido utilizadas para avaliar a qualidade dos alimentos e os efeitos nutricionais dessa fração para ruminantes e monogástricos (JERACI e VAN SOEST, 1990; VAN SOEST et al. 1991; ANNISON e CHOCT, 1994; WARPECHOWSKI, 1996; GINGER-REVERDIN et al. 2002).

ANNISON e CHOCT (1994) afirmam que a capacidade de hidratação é uma característica tanto da fibra solúvel quanto da insolúvel. Segundo VAN SOEST, (1994), essa capacidade, no caso da fibra insolúvel, depende da presença de grupos hidrofílicos, da área de superfície da molécula da fibra, do espaço livre na estrutura intermolecular e, consequentemente, do tamanho de partícula, e está correlacionada com a capacidade tamponante.

Para VAN SOEST et al. (1991), a capacidade tamponante é a facilidade de uma molécula reter ou trocar cátions por $\mathrm{H}^{+}$, conforme o $\mathrm{pH}$ do meio onde ela se encontra. Trabalhos como o de McCONNEL et al. (1974) mostram que essa propriedade é inerente à matriz da parede celular de cada alimento. McBURNEY et al. (1983) descreveram metodologia específica para a determinação da capacidade tamponante na fibra em 
Propriedades físico-químicas da fibra de diferentes palhas de trigo em detergente neutro

detergente neutro de alimentos, baseada na titulação direta de mistura aquosa da fibra com solução de ácido clorídrico. Entretanto, de acordo com a classificação utilizada por GINGER-REVERDIN et al. (2002), a medida obtida com a metodologia descrita por McBURNEY et al. (1983) determina a acidez titulável, que é definida como a quantidade de ácido requerida para diminuir uma unidade no $\mathrm{pH}$ da solução, com os resultados expressos em $\mathrm{mEq}$ de ácido/g de matéria seca (MS) do alimento. A capacidade tamponante pode ser então calculada dividindo os valores de acidez titulável pelo intervalo de $\mathrm{pH}$ considerado na determinação.

Este trabalho teve como objetivo caracterizar a fibra em detergente neutro da palha de diferentes variedades de trigo quanto às propriedades físico-químicas de capacidade tamponante e capacidade de hidratação.

\section{Material e Métodos}

O material experimental foi constituído por cinco palhas de trigo, incluindo quatro variedades: BR-23A (proveniente de uma lavoura em Carazinho/RS, em 1993), BR-23B, BR-35, CEP-21 e CEP-19 (provenientes de uma única safra do Centro Nacional de Pesquisa do Trigo - EMBRAPA, de Passo Fundo, RS, em 1992).

A preparação da fibra das palhas de trigo para as análises e a capacidade tamponante destas preparações foi feita segundo metodologia adotada por McBURNEY et al. (1983), no presente trabalho, chamada de acidez titulável. Os resultados de acidez titulável foram expressos em mmol de $\mathrm{HCl}$ adicionados por $100 \mathrm{~g}$ de matéria seca da preparação de fibra e em função dos valores de $\mathrm{pH}$ obtidos em cada ponto. Assim, foram obtidas as curvas de titulação das preparações de fibra e determinadas duas medidas: a quantidade de $\mathrm{HCl}$ necessária para levar o $\mathrm{pH}$ a 2,00 e a quantidade gasta no intervalo de $\mathrm{pH}$ em que houve a maior variação entre curvas, seguindo as indicações de McBURNEY et al. (1983).
A capacidade de hidratação da preparação de fibra das palhas de trigo foi determinada conforme a técnica descrita por McCONNELL et al. (1974), com as seguintes modificações: $0,5 \mathrm{~g}$ da preparação de fibra foram colocadas em tubos de centrífuga (PVC, $50 \mathrm{ml}$ ) com $25 \mathrm{~mL}$ de $\mathrm{H}_{2} \mathrm{O}$ destilada e deionizada, tampadas e mantidas em banho-maria de $36-38^{\circ} \mathrm{C}$ com agitação por vinte e quatro horas. Então o material foi centrifugado a $14.000 \times \mathrm{xg}\left(20^{\circ} \mathrm{C}\right)$ por 60 minutos, filtrado a vácuo em funil com filtro de porosidade grossa e as preparações de fibras úmidas passadas para cadinhos tarados de porcelana e pesadas. Os resultados foram calculados como gramas de água por grama de matéria seca da preparação de fibra.

Foram feitas determinações de acordo com a metodologia descrita por GOERING e VAN SOEST (1970) para fibra em detergente neutro (FDN), fibra em detergente ácido, lignina, matéria seca a $105^{\circ} \mathrm{C}$ e cinzas a $550^{\circ} \mathrm{C}$ nas palhas e de matéria seca e FDN nas preparações de fibra das palhas.

Utilizou-se o delineamento experimental em blocos casualizados, com seis repetições em cada tratamento, sendo utilizados seis blocos (corridas) para a capacidade tamponante e três para a capacidade de hidratação. Os resultados foram submetidos a uma análise de variância, e as médias foram comparadas pelo teste de Tukey. Também foram calculados e analisados os coeficientes de correlação linear entre as variáveis estudadas.

\section{Resultados e Discussão}

$\mathrm{Na}$ TABELA 1 são apresentados os valores da composição química média das palhas e os teores de FDN das preparações de fibra. Os resultados médios foram de 81,25\% FDN, 26,31\% hemicelulose, 7,72\% lignina e 47,22\% celulose, com diferenças em torno de $10 \%$ para todos os valores, exceto para a lignina, maior que $20 \%$. Para todas as preparações de fibra observouse mais de $99 \%$ de FDN.

TABELA 1 - COMPOSIÇÃO QUÍMICA PERCENTUAL DAS PALHAS DE TRIGO E TEORES DAS PREPARAÇÕES DE FIBRA.

\begin{tabular}{lccccccc}
\hline Variedade $^{1}$ & $\mathrm{MS}^{2}$ & $\mathrm{MO}^{3}$ & $\mathrm{FDN}^{4}$ & hemicelulose & lignina & celulose & FDNP $^{5}$ \\
\hline BR-23A & 89.51 & 95.12 & 84.17 & 27.61 & 8.29 & 48.27 & 99.17 \\
CEP-19 & 88.81 & 95.36 & 79.93 & 25.04 & 8.45 & 46.44 & 99.13 \\
CEP-21 & 88.56 & 95.27 & 80.09 & 27.46 & 6.75 & 45.88 & 99.29 \\
BR-23B & 89.15 & 95.46 & 80.49 & 25.91 & 7.11 & 47.47 & 99.02 \\
BR-35 & 89.10 & 95.68 & 81.56 & 25.52 & 7.98 & 48.06 & 99.35 \\
\hline
\end{tabular}

${ }^{1}$ As letras indicam diferentes safras e locais de cultivo; ${ }^{2}$ Matéria seca; ${ }^{3}$ Matéria orgânica; ${ }^{4} \mathrm{Fibra}$ em detergente neutro; ${ }^{5}$ Valores de fibra em detergente neutro das preparações de fibra das palhas de trigo

Na FIGURA 1, em que estão representadas as curvas médias de titulação das preparações de fibra, observase uma maior variação no intervalo de $\mathrm{pH}$ de 5,36 a 2,74 . A acidez titulável neste intervalo (ATi) foi significativamente maior para BR-23, depois BR-35 e CEP-19 e menor para CEP-19, e BR-23A (TABELA 2). Para a $A T$, os valores observados para $B R-23 B, B R-$
35 e CEP-19 foram significativamente maiores que para as outras palhas, que não diferiram estatisticamente entre si (TABELA 2). Houve diferença significativa $(P<0,05)$ entre corridas para a ATi, o que não ocorreu para a AT, e os coeficientes de variação foram 7,07 e $4,58 \%$, respectivamente.

Para capacidade de hidratação, não se detectou 
diferença significativa entre as amostras, observando-se uma retenção média de $5,34 \mathrm{~g} \mathrm{H}_{2} \mathrm{O}$ / $\mathrm{g}$ de matéria seca da preparação de fibra (TABELA 2). JERACI e VAN SOEST (1990), utilizando palha de arroz contendo $70,2 \%$ de FDN e com tamanho médio de partícula de $0,5 \mathrm{~cm}$, observaram $\mathrm{CH}$ de
$8,6 \mathrm{~g} \mathrm{H}_{2} \mathrm{O} / \mathrm{g} \mathrm{MS}$. Entretanto, de acordo com esses mesmos autores, a moagem fina resulta em perda de espaço interior, com aumento de densidade da partícula e diminuição na capacidade de hidratação, o que poderia explicar os menores valores no presente trabalho.

FIGURA 1 - CURVAS DE TITULAÇÃO DA FIBRA EM DETERGENTE NEUTRO DE DIFERENTES PALHAS DE TRIGO. LETRAS DISTINTAS JUNTO AO NOME DA VARIEDADE DE TRIGO INDICAM DIFERENTES SAFRAS E LOCAIS DE CULTIVO.

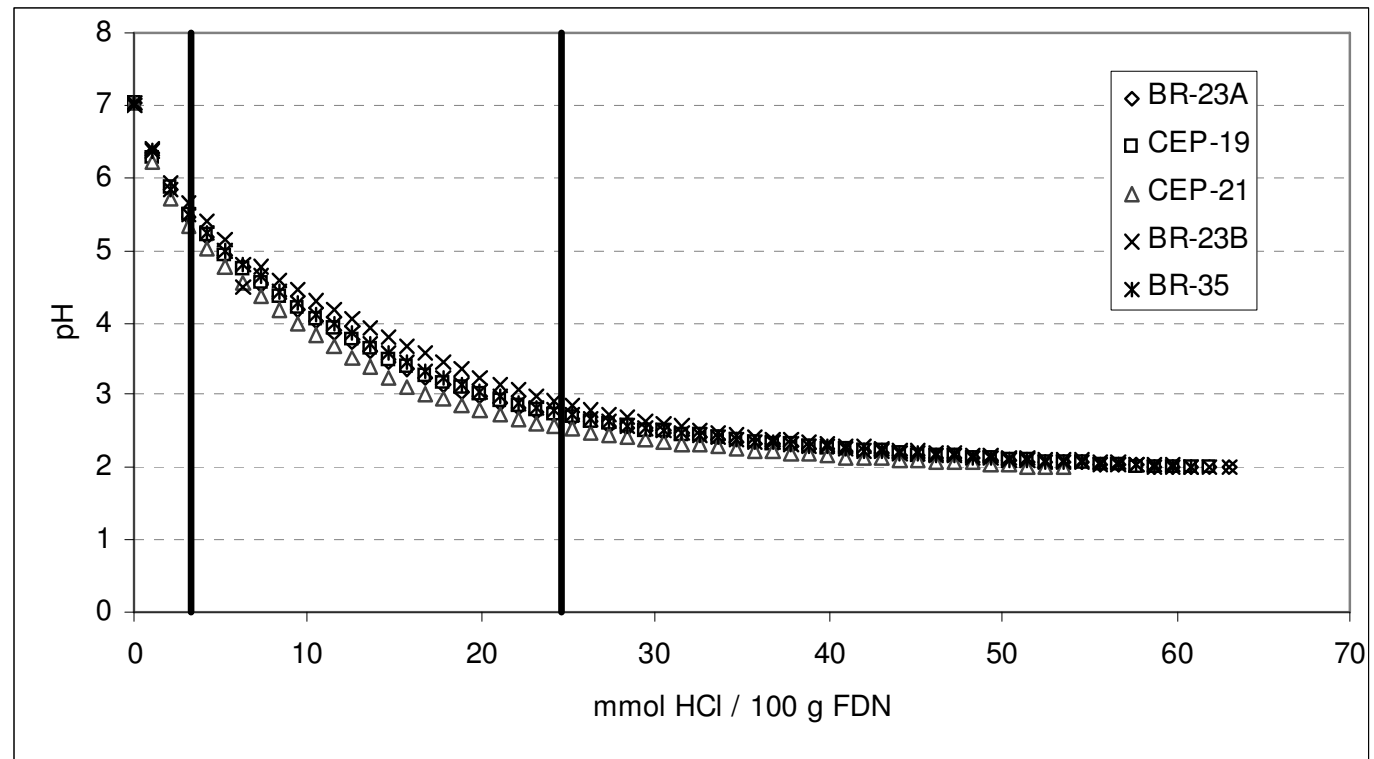

TABELA 2 - ACIDEZ TITULÁVEL (AT), ACIDEZ TITULÁVEL NO INTERVALO DE pH (ATi) E CAPACIDADE DE HIDRATAÇÃO (CH) DA FIBRA EM DETERGENTE NEUTRO DE DIFERENTES PALHAS DE TRIGO.

\begin{tabular}{lccc}
\hline Variedades $^{1}$ & \multicolumn{1}{c}{ AT } & ATi & CH \\
\hline BR-23A & \multicolumn{2}{c}{$\mathrm{mmol} \mathrm{HCl} / 100 \mathrm{~g} \mathrm{MS}$} & $\mathrm{g} \mathrm{H} 2 \mathrm{O} / \mathrm{g} \mathrm{MS}$ \\
CEP-19 & $53.37 \mathrm{~b}$ & $13.83 \mathrm{c}$ & $5.27 \mathrm{a}$ \\
CEP-21 & $59.33 \mathrm{a}$ & $17.18 \mathrm{~b}$ & $5.50 \mathrm{a}$ \\
BR-23B & $52.18 \mathrm{~b}$ & $15.05 \mathrm{c}$ & $5.07 \mathrm{a}$ \\
BR-35 & $61.24 \mathrm{a}$ & $19.60 \mathrm{a}$ & $5.46 \mathrm{a}$ \\
\hline
\end{tabular}

Médias seguidas de letras distintas na coluna diferem significativamente pelo teste de Tukey $(P<0.05)$. ${ }^{1}$ As letras junto ao nome da variedade indicam diferentes safras e locais de cultivo.

O trabalho de VAN SOEST et al. (1991), mostrou que propriedades físico-químicas como a capacidade de hidratação e a capacidade tamponante estão correlacionadas com um coeficiente de correlação próximo a 0,70. Confirmando essa afirmação, os resultados do presente trabalho demonstraram coeficientes de correlação linear de 0,69 entre a ATi e a $\mathrm{CH}$ e de 0,88 entre a AT e a $\mathrm{CH}$ (TABELA 3). 
Propriedades físico-químicas da fibra de diferentes palhas de trigo em detergente neutro

TABELA 3 - COEFICIENTES DE CORRELAÇÃO LINEAR ENTRE AS VARIÁVEIS ESTUDADAS E OS RESPECTIVOS NÍVEIS DE SIGNIFICÂNCIA.

\begin{tabular}{ccc}
\hline Variáveis $^{1}$ & $r$ & $\mathrm{P}>\mathrm{t}$ \\
\hline $\mathrm{AT} \times \mathrm{ATi}$ & 0,924 & 0,023 \\
$\mathrm{AT} \times \mathrm{CH}$ & 0,882 & 0,046 \\
$\mathrm{ATi} \times \mathrm{CH}$ & 0,694 & 0,193 \\
\hline
\end{tabular}

${ }^{1} \mathrm{AT}$, acidez titulável das preparações de fibra (mmol/100g); ATi, acidez titulável no intervalo de maior variação entre as fibras; $\mathrm{CH}$, capacidade de hidratação das preparações de fibra $(\mathrm{g} / \mathrm{g})$.

\section{Conclusões}

Nas condições do presente trabalho pode-se concluir que diferentes palhas de trigo diferem quanto à capacidade tamponante mas não quanto à capacidade de hidratação; que as diferenças observadas são devidas tanto à variedade quanto à safra e/ou local de plantio do trigo; e que as medidas acidez titulável e capacidade de hidratação da fibra em detergente neutro dos materiais estudados se correlacionam positivamente.

\section{Referências}

ANNISON, G.; CHOCT, M. Plant polysaccharides - their physiochemical properties and nutritional roles in monogastric animals. In: LYONS, T.P.; JACQUES, K. A. Biotechnology in the feed Industry. Procedings of Alltech's Tenth Annual Symposium. Nottingham: Nottingham University Press, 1994, p.51-66.

FERREIRA, W.M. Os componentes da parede celular vegetal na nutrição de não ruminantes. In: SIMPÓSIO INTERNACIONAL DE PRODUÇÃO DE NÃO RUMINANTES. Reunião Anual da Sociedade Brasileira de Zootecnia, XXXI. 1994, Maringá, Anais da XXXI Reunião Anual da Sociedade Brasileira de Zootecnia. Maringá: SBZ, 1994. p.85-113.

GINGER-REVERDIN, S.; DUVAUX-PONTER, C.; SAUVANT, D.; MARTIN, O.; PRADO, I.N.; MÜLER, R. Intrinsic buffering capacity of feedstuffs. Animal Feed Science and Tecnology. Amsterdam, v.96, p.83-102, 2002.

GOERING, H.G. \& VAN SOEST, P.J. Forage fiber analysis (apparatus, reagents, procedures and some applications). In: Agriculture Handbook n³79, USDA. Washington, DC. 1970.
JERACI, J. L.; VAN SOEST, P. J. Improved methods for analysis and biological characterization of fiber. Advances in Experimental Medicine and Biology. New York, v.270, p.245-263. 1990.

JERACI, J.L.; HORVATH, P.S. In vitro fermentation of dietary fiber by human fecal organisms. Animal Feed Science and Technology. Amsterdam, v.23, p.121-140, 1989.

McBURNEY, M. I.; VAN SOEST, P. J.; CHASE, L. E. Cation exchange capacity and buffering capacity of neutral-detergent fibres. Journal of the Science of Food and Agriculture. Mysore, v.34, p.910-916, 1983.

McCONNELL, A.A.; EASTWOOD, M.A.; MITCHELL, W.D. Physical characteristics of vegetable foodstuffs that could influence bowel function. Journal of the Science of Food and Agriculture. Mysore, v.25, p.1457-1464, 1974.

VAN SOEST, P. J. Nutritional ecology of the ruminant. Ithaca: Cornell University Press, 1994. 476 p.

VAN SOEST, P. J.; ROBERTSON, J. B.; LEWIS, B. A. Methods for dietary fiber, neutral detergent fiber and nonstarch polysaccharides in relation to animal nutrition. Journal of Dairy Science. Savoy, v.74, p.3583-3597. 1991.

WARPECHOWSKI, M. B. Efeito da fibra insolúvel da dieta sobre a passagem no trato gastrintestinal de matrizes machos pesados, intactos, cecectomizados e fistulados no íleo terminal. Porto Alegre, 1996. Dissertação (Mestrado em Zootecnia), Programa de Pós Graduação em Zootecnia, Faculdade de Agronomia da Universidade Federal do Rio Grande do Sul.

Recebido para publicação:

$16 / 11 / 2005$

Aprovado: 